\title{
Control and Data Acquisition System of Electron Accelerator for Radiation Processing
}

\author{
Wiesław Maciszewski, Janusz Łukaszewicz, \\ Wojciech Migdal, Andrzcj G. Chmielewski \\ Institute of Nuclear Chemistry and Technology \\ 03-195 Warsaw, Dorodna 16, Poland
}

\begin{abstract}
In the INCT a Pilot Plant for Food Irradiation started the operation November 1992. The plant is equipped with linear electron accelerator. To improve the operation it was decided to equip the accelerator with computerized system of the control and data acquisition. It is based on IBM PC/AT computer and the BITBUS interconnection system.
\end{abstract}

\section{INTRODUCTION}

In the Pilot Plant for Food Irradiation, new accelerator facility is installed. This is Russian made industrial electron linac "Elektronika 10-10" with electron energy of $10 \mathrm{MeV}$ and mean power of the beam up to $10 \mathrm{~kW}$ [1]. The main parts of the accelerator are RF magnetron power generator, travelling wave type accelerating structure, high power pulse modulator, an electron gun and control system. To assure more effective operation of the accelerator and for its more convenient inspection, it was decided to equip the accelerator with computerized system of the control and data acquisition. Such a system will be very useful for the assurance of the irradiation process quality. The system is based on IBM PC/AT computer and the BITBUS interconnection system posessing distributed control modules $[2,3]$.

\section{REQUIREMENTS FOR THE SYSTEM}

The task of the system on its first stage of realization was supervising selected parameters of the accelerator, and memorizing/visualizing their current values in the form convenient for inspection of the accelerator operation by the operator. The second stage will be full automatization of accelerator operation. After realization of the second stage of the system we will be able to assure full automation of accelerator operation including the reaching selected parameters of the accelerator and their stabilization with a closed feedback loop. To do it, considerable modification of electronic systems of accelerator is needed. Starting the second stage of realization of the system is planned to be done after finishing the warranty period for the accelerator. To attain practical realization of the system sixteen analog signals taken from the electronic circuits of the acceleartor should be processed. The list of signals to be processed is as follows:

1 - straight beam current

2 - bended beam current

3 - bending magnet current

4 - scanning magnet current

5 - mean magnetron current

6 - magnetron voltage

7 - magnetron frequency deviation

8 - HF power level

9 - magnetron solenoid current

10 - accelerator solenoid current, section I

11 - accelerator solenoid current, section II

12 - accclcrator solcnoid current, section III

13 - gun voltage

14 - velocity of the conveyor

15 - vacuum level

16 - mains voltage

\section{SYSTEM DESCRIPTION}

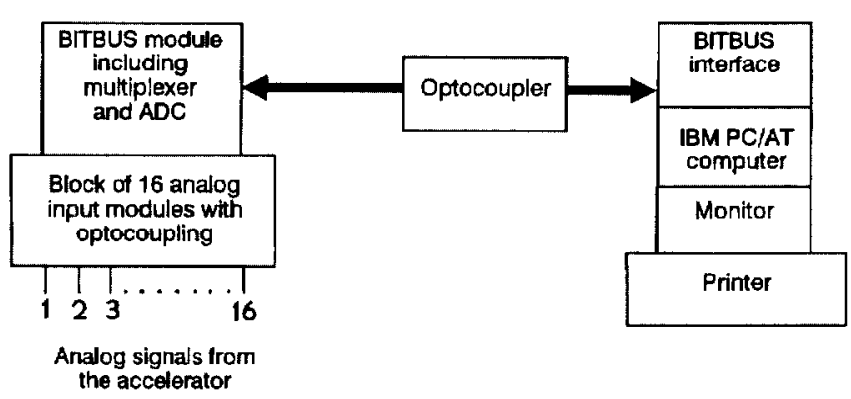

Fig.1. Block diagram of the system of data acquisition 
The block diagram of the system of data acquisition is shown in fig. 1.

Analog signals from the accelerator are delivered to the block of sixteen analog modules with optoisolation. Next, these signals are fed to the BITBUS analog module which includes multiplexer and analog-to-digital converter.This module realizes sequential ADC conversion of the signals and their preliminary processing. Digital signals are transmitted to the computer by means of BITBUS data way. Operation of the system is based on appropriate software, which may be divided into three following parts:

1 - local software of analog module responsible for carrying out the measurements, their results memorizing and preliminary processing inside the module;

2 - data base, which ensures to storage operational parameters of the accelerator and various data of the plant's activity, for example registration of customers, write out the cheques and certificates of radiation treatment. Apart from this, data base allows to define all parameters of measuring channels, which gives the operator the possibility of adjusting the voltage on a given measuring output and related physical parameter of the accelerator;

3 - controlling software, written in $\mathrm{C}$ language, which allows the current inspection of the accelerator parameters and directs the transmission of information trough the BITBUS data way, participating in data exchange between analog modulc and data base.

\section{CONCLUSIONS}

The system of data acquisition (stage 1) has been connected to the "Elektronika 10-10" rf linac and preliminarily tested. In consequence considerable improvement in the inspection of the accelerator was obtained.

Further development of the system (stage 2) for the extention its ability in the control and stabilization of important accelerator parameters is under consideration.

\section{REFERENCES}

[1] Linear Electron Accelerator "Elektronika 10-10", Tech nical Description, Moscow (1991).

[2] The BITBUS Interconnect Serial Control Bus Specification, Intel Corp., order number 280645-001 (1988).

[3] Distributed Control Modules, Intel Corp., order number 230973-004 (1988). 